\title{
Téoros
}

Revue de recherche en tourisme

\section{Développement local durable et tourisme au Honduras}

\section{Paul Bodson et Jorge Guerrero}

Volume 27, numéro 3, automne 2008

URI : https://id.erudit.org/iderudit/1070789ar

DOI : https://doi.org/10.7202/1070789ar

Aller au sommaire du numéro

Éditeur(s)

Université du Québec à Montréal

ISSN

0712-8657 (imprimé)

1923-2705 (numérique)

Découvrir la revue

Citer cette note

Bodson, P. \& Guerrero, J. (2008). Développement local durable et tourisme au Honduras. Téoros, 27(3), 89-95. https://doi.org/10.7202/1070789ar d'utilisation que vous pouvez consulter en ligne.

https://apropos.erudit.org/fr/usagers/politique-dutilisation/ 


\section{Chroniques -}

Centreinternational de formation et derecherche en tourisme

\section{Développement local durable et tourisme au Honduras'}

\section{Paul Bodson et Jorge Guerrero}

Mers cristallines, îles paradisiaques, plages soyeuses, montagnes verdoyantes couvertes de pins majestueux, forêts tropicales, biodiversité, patrimoine précolombien et colonial, diversité ethnique et culturelle, un coin d'Amazonie dans l'hémisphère nord, le Honduras semble voué à un destin touristique hors du commun. En même temps, le Honduras figure, au même titre que le Nicaragua et Haïti, sur la liste des pays les plus pauvres d'Amérique. Si l'on se réfère à l'indice de développement humain (IDH) publié par le Programme des Nations Unies pour le développement (PNUD) (rapport 2007-2008), le pays se situe au $115^{\mathrm{e}}$ rang parmi les 177 pays considérés.

Dans une perspective temporelle, la situation du pays apparaît tout aussi critique. Le Honduras n'a pas enregistré de réduction vraiment significative du niveau de pauvreté durant les dernières années et ce, en dépit d'une croissance économique relativement soutenue (3,8 \% annuellement de 1990 à 2005). L'évolution actuelle de l'économie diffuse aussi le développement de façon très inégale au niveau de certains groupes ou communautés, en particulier les femmes, les autochtones, les communautés isolées, et aboutit à consolider des disparités de plus en plus insupportables. Les régions et les communautés locales affectées par la pauvreté disposent pourtant le plus souvent de potentiels inexploités. $Y$ manquent un certain nombre de conditions requises à leur mise en valeur. II est dès lors impérieux pour les groupes et les communautés particulièrement vulnérables aux problèmes de pauvreté et d'inégalités sociales d'identifier leurs ressources et de les valoriser de manière à soutenir leur propre développement et leur épanouissement. La mise en place d'une dynamique de développement articulée sur des initiatives et des projets locaux exige par contre que soient réunies un certain nombre de conditions favorables. Des travaux et des expériences menés en Amérique centrale et au Mexique en matière de développement régional et local (PNUD, Banque mondiale, agences de coopération internationale, Organisation Internationale du Travail, Facultad Latino Americana de Ciencias Sociales) ressortent un certain nombre de constats et de conclusions incontournables dans la formulation de projets d'appui au développement local :
- Les communautés locales défavorisées sont systématiquement aux prises avec un besoin urgent de formation de base et de formation spécialisée.

- Les projets ponctuels sont susceptibles d'avoir un impact renforcé et catalyseur sur le développement s'ils sont associés à une prise en charge globale de la région où ils s'insèrent.

- Le développement d'une région suppose une certaine organisation, en particulier des infrastructures et des services, y compris des services sociaux.

- Pour prendre consistance, cette organisation a besoin de s'ancrer dans un processus de prise de conscience communautaire associant des mécanismes continus de consultation, de concertation et un soutien d'accompagnement par des groupes communautaires (généralement de type "organisation non gouvernementale») bien insérés dans le milieu.

- Pour prendre son essor, le développement régional ou communautaire doit s'inscrire dans une démarche à moyen terme attentive à réajuster, à renouveler et à diversifier les projets en cours, les sources de financement requises et à consolider la formation et le suivi d'accompagnement.

Afin de promouvoir un développement durable, en particulier dans les régions structurellement marginalisées par la pauvreté, le Honduras s'est doté d'un cadre politique destiné à mobiliser de façon concertée les énergies du pays et les interventions des banques de développement et de la coopération internationale ("Stratégie pour la réduction de la pauvreté", "Programme national de décentralisation et de développement local » et mises à jour successives). Tous les secteurs d'activité économique, en particulier le secteur du tourisme, sont conviés à s'impliquer dans l'effort de lutte pour la réduction de la pauvreté.

Pour développer le tourisme, le Honduras dispose d'avantages comparatifs indéniables (vestiges préhispaniques, patrimoine colonial, multiculturalisme, diversité de ressources naturelles, plages, muraille de corail au large de la côte des Caraïbes...). Mais ces atouts restent relativement sous-exploités. 
Tableau 1

Potentiels touristiques des pays d'Amérique centrale

\begin{tabular}{|c|c|c|c|c|c|c|}
\hline & $\begin{array}{c}\text { Vestiges } \\
\text { préhispaniques }\end{array}$ & $\begin{array}{l}\text { Diversité } \\
\text { culturelle }\end{array}$ & Plages & Snorkeling & Nature & $\begin{array}{c}\text { Patrimoine } \\
\text { culturel colonial }\end{array}$ \\
\hline Honduras & $\sqrt{ }$ & $\sqrt{ }$ & $\sqrt{ }$ & $\sqrt{ }$ & $\sqrt{ }$ & $\sqrt{ }$ \\
\hline Costa Rica & & & $\sqrt{ }$ & & $\sqrt{ }$ & \\
\hline Guatemala & $\sqrt{ }$ & $\sqrt{ }$ & & & $\sqrt{ }$ & $\sqrt{ }$ \\
\hline Belize & $\sqrt{ }$ & $\sqrt{ }$ & $\sqrt{ }$ & $\sqrt{ }$ & $\sqrt{ }$ & \\
\hline Salvador & $\sqrt{ }$ & & $\sqrt{ }$ & & & \\
\hline Nicaragua & $\sqrt{ }$ & $\sqrt{ }$ & $\sqrt{ }$ & & $\sqrt{ }$ & $\sqrt{ }$ \\
\hline Panama & $\sqrt{ }$ & $\sqrt{ }$ & $\sqrt{ }$ & & $\sqrt{ }$ & $\sqrt{ }$ \\
\hline
\end{tabular}

Le Honduras s'est dès lors doté d'un plan de développement du tourisme dont la mise en œuvre prévue s'étend à l'horizon 2021 ( SStratégie Nationale de développement durable du secteur du tourisme au Honduras»). La préoccupation de la lutte pour la réduction de la pauvreté est sous-jacente à son articulation. L'accent est mis prioritairement sur le développement de zones à forte attraction touristique (tourisme international et tourisme national) capables de générer un apport important de revenus en particulier sous forme de devises et d'entraîner dans son sillage la création de nombreux emplois (îles de Bahia, Tela et Trujillo sur la côte des Caraïbes, Copán). Complémentairement aux méga projets, le plan de développement du tourisme entend promouvoir un ensemble de projets de petite ou moyenne dimension (golfe de Fonseca, corridor Omoa-Cortés, Ruta Lenca, corridor archéologique Los Naranjos, el Puente et Copán, villes coloniales). Certains de ces projets concernent directement des populations parmi les plus démunies du Honduras.

Conscient de la diversité de son potentiel touristique, mais aussi du besoin des populations d'améliorer leurs conditions de vie en harmonie avec leur ancrage territorial, le Honduras s'est de plus doté d'instruments complémentaires destinés à orienter la mise en place d'activités dans certains volets spécifiques du tourisme. Le pays figure officiellement sur la liste des pays promoteurs du "géotourisme». Dans la foulée de l'évolution des tendances actuelles du tourisme, il entend aussi faire de l'écotourisme une dimension importante de son développement touristique.

L'Institut hondurien du tourisme $(\mathrm{IHT})$, sous la direction du ministre du Tourisme, sert de plaque tournante pour canaliser la mise en œuvre pratique de ces orientations et coordonner les implications des banques de développement et des agences de coopération internationale.
Qu'en est-il dans les faits? Dans quelle mesure le tourisme au Honduras s'inscrit-il dans les perspectives d'un autre tourisme riche, pour «l'accueilli », de situations et d'expériences l'ouvrant à un monde qui le déconnecte du vécu quotidien, mais en même temps respectueux de "l'accueillant », de son mode de vie, de son environnement, du rythme de son évolution dans la société actuelle et capable de contribuer à répondre à ses besoins et à ses attentes, en particulier en matière de développement?

Lorsqu'il s'implante dans une région, le tourisme a généralement tendance à se cristalliser autour de pôles majeurs d'attraction (par exemple, au Honduras, les îles ou la côte des Caraïbes ou encore Copán Ruinas). II y module son organisation de manière à répondre aux attentes de la clientèle tout en assurant les meilleures marges de profit à ses promoteurs. II en résulte très souvent un développement du tourisme en vase clos, profitant à un nombre restreint de personnes, d'entreprises ou d'organisations, mais peu soucieux de l'environnement et du bien-être des populations locales. Mis à part les personnes directement impliquées dans les pôles touristiques, les retombées pour l'ensemble de la population locale sont souvent très faibles. Dans la population locale, néanmoins, d'aucuns essayeront de tirer profit des opportunités latentes qu'offre la venue de touristes dans leur région. Des projets divers émergeront. Ils seront généralement de petite dimension, caractérisés par un manque de ressources et un fonctionnement de type «informel». En dépit des efforts déployés, ce genre de projet est souvent voué à un avenir très incertain, sauf quelques cas récupérés par le tourisme dominant pour rejoindre certaines attentes particulières de la clientèle de passage.

Le «modèle » risque par contre d'être progressivement remis en question en raison de priorités provenant des pouvoirs publics et d'instances internationales, en raison de la prise de conscience de plus en plus forte des implications sociales du tourisme et de ses répercussions sur l'environnement, mais aussi en raison 


\section{Illustration 1}

\section{Arrivées de touristes en Amérique centrale, 2006}

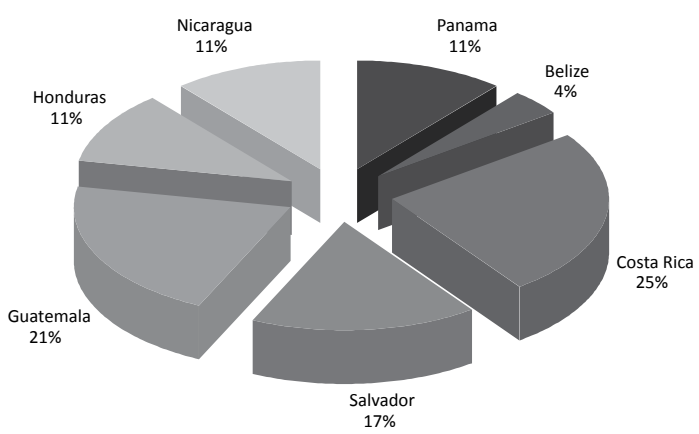

Source : Sistema de la Integración Centroamericano, SICA.

de l'attrait croissant chez le touriste pour des expériences le rapprochant des populations locales, de leur vécu et de leur relation à l'environnement.

Par contre, associer le tourisme à une dynamique de développement local pose de toute évidence de sérieux défis.

Pour attirer une masse critique de touristes qui puisse avoir un impact sur le développement local, une région doit disposer d'un pôle d'attraction. C'est le cas, par exemple, des ruines mayas à Copán ou encore de Tela et les environs sur la côte des Caraïbes. À défaut, un ensemble diversifié de potentiels touristiques peut aussi constituer la base d'un pôle d'attraction. On peut penser ici à la Ruta Lenca ou encore à la Ruta Moskitia.

Le pôle d'attraction doit pouvoir servir de catalyseur, de manière à susciter l'émergence d'initiatives locales et en favoriser l'accès aux touristes en visite dans la région. Ces initiatives ne sont possibles qu'avec la participation des populations locales. Ces dernières sont généralement très accueillantes vis-à-vis des visiteurs, tant nationaux qu'étrangers. Un pas supplémentaire est cependant indispensable pour le développement d'un tourisme local. II faut que les populations locales soient favorables au tourisme parce qu'elles y trouvent leur propre intérêt, à partir de leurs conditions et de leurs modes de vie et qu'elles s'impliquent activement dans le développement d'initiatives locales en tourisme.

Laissées à elles-mêmes, de nombreuses initiatives locales risquent de se confiner à des réalisations peu propices à leur insertion dans le tourisme contemporain. Les communautés locales défavorisées en fait se heurtent systématiquement à un besoin urgent de formation dans la conception, la formalisation, la mise en œuvre et le suivi de projets tablant sur les potentiels des communautés locales. Corrélativement, le manque de capital mine le développement de nombreuses initiatives locales.

\section{Tableau 2}

Revenus du tourisme en Amérique Centrale (millions USD)

\begin{tabular}{lccccccc} 
Pays & $\mathbf{2 0 0 0}$ & $\mathbf{2 0 0 1}$ & $\mathbf{2 0 0 2}$ & $\mathbf{2 0 0 3}$ & $\mathbf{2 0 0 4}$ & $\mathbf{2 0 0 5}$ & $\mathbf{2 0 0 6}$ \\
\hline Belize & 120 & 121 & 133 & 156 & 173 & 175 & 166 \\
\hline Costa Rica & 1229 & 1278 & 1078 & 1199 & 1343 & 1551 & 1664 \\
\hline Salvador & 254 & 235 & 342 & 373 & 425 & 644 & 871 \\
\hline Guatemala & 419 & 493 & 612 & 600 & 770 & 869 & 1012 \\
\hline Honduras & 262 & 275 & 342 & 363 & 391 & 408 & 490 \\
\hline Nicaragua & 111 & 109 & 107 & 152 & 167 & 207 & 239 \\
\hline Panama & 576 & 626 & 679 & 805 & 906 & 971 & 1270 \\
\hline
\end{tabular}

Source : Sistema de la Integración Centroamericano, SICA.

Vu le type de services dans lesquels elles se spécialisent, les initiatives locales se doivent aussi, pour survivre et se développer, de s'associer à des réseaux qui leur permettent de canaliser vers elles les flux de touristes qui fréquentent leur région.

La mise en valeur des potentiels touristiques locaux et des projets qui leur sont associés suppose aussi des infrastructures de base. Celles-ci concernent à la fois l'accessibilité physique, les conditions d'accueil (hébergement, alimentation...), les services de santé, la sécurité, les réseaux d'information y compris l'information virtuelle.

Même adéquatement développées, les activités du tourisme comportent des risques de se refermer sur elles-mêmes. II reviendra aux autorités politiques nationales et locales d'orienter les retombées économiques du tourisme, de manière à améliorer les conditions de vie des populations locales.

Les régions du Honduras, en particulier les zones touchées par des niveaux de grande pauvreté structurelle, rencontrent très difficilement l'ensemble des conditions évoquées. Le ministère du Tourisme (IHT), les organismes internationaux (Banque mondiale, Banque Interaméricaine de Développement, agences de coopération), de nombreuses organisations non gouvernementales (ONG) nationales et internationales interviennent dès lors, généralement en association ou en concertation, pour consolider progressivement les bases d'un tourisme servant de levier au développement local.

LeprojetPartenariatUniversitaireen CoopérationetDéveloppement (PUCD volet 2), Développement Local et Tourisme, 2003-2009, financé par l'Agence canadienne de développement international (ACDI) (gestion du programme PUCD volet 2 effectuée par l'Association des universités et collèges du Canada), s'inscrit aussi dans cette ligne. Un accord de collaboration entre I'Université 
Positionnement du projet dans la problématique associant le tourisme au développement local en régions structurellement paupérisées au Honduras

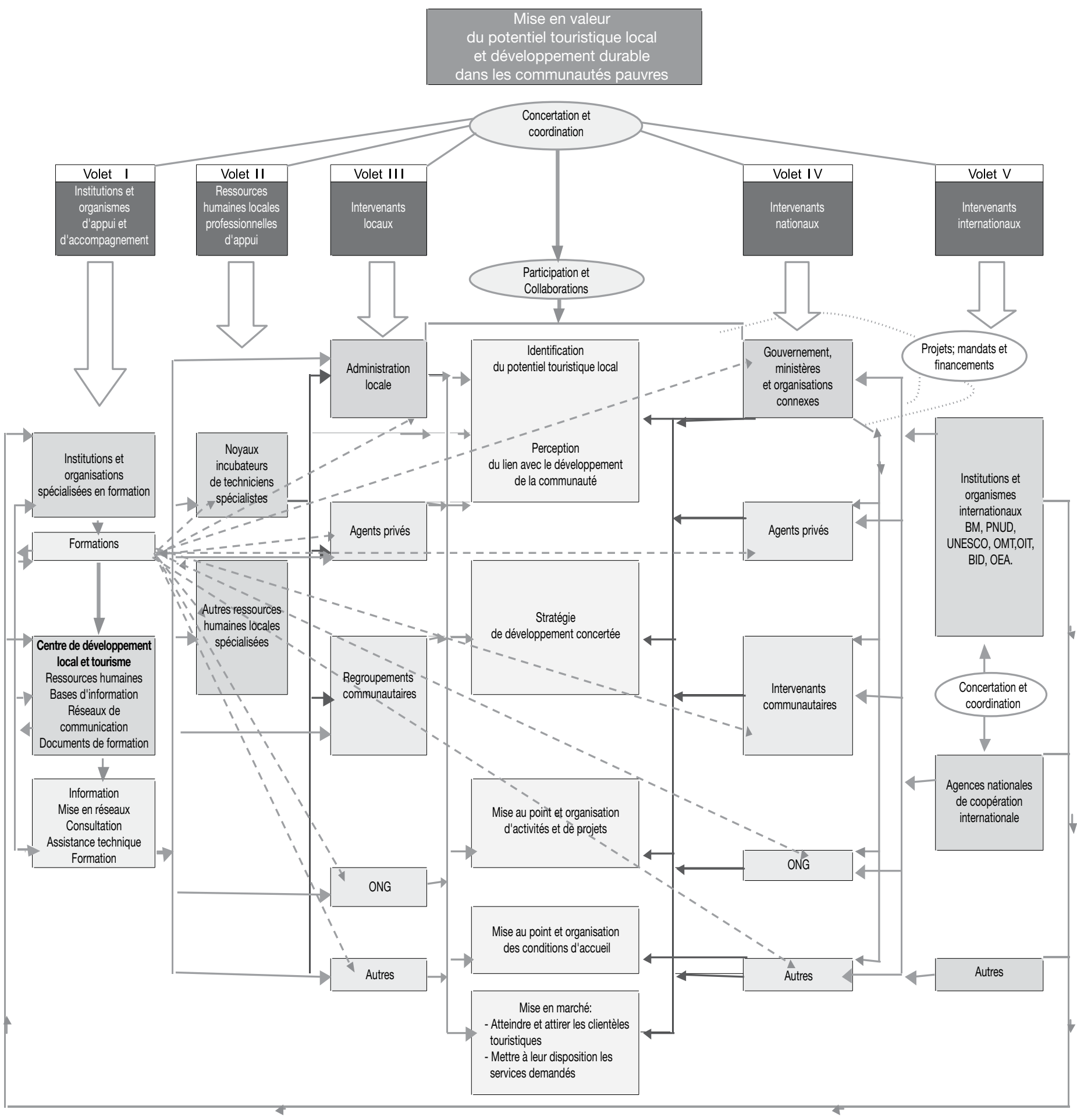

Source : PUCD volet 2, Développement local durable et tourisme au Honduras. 
du Québec à Montréal (UQAM) et la Universidad Tecnológica Centroamericana (UNITEC) au Honduras sert de pierre angulaire à la mise en œuvre du projet. Compte tenu des rôles et des interventions de l'Institut hondurien du tourisme, des organismes et des agences internationaux et locaux et des spécificités du programme PUCD volet 2, compte tenu par ailleurs des priorités qui s'imposent à l'échelle locale dans une perspective de développement à moyen et long terme, le projet a concentré ses efforts sur la formation de ressources humaines capables d'intervenir en matière de tourisme dans les régions structurellement marginalisées par la pauvreté.

L'illustration 2 synthétise la logique du positionnement du projet dans la problématique associant le tourisme au développement local en régions structurellement paupérisées au Honduras.

La mise en œuvre du projet comporte deux volets principaux. Le premier concerne directement la formation et la recherche, le second l'infrastructure de soutien. Afin d'assurer à l'ensemble de la démarche son ancrage dans la réalité, deux zones cibles de travail sur le terrain ont été sélectionnées : la couronne est/sud-est de Tegucigalpa et, dans l'ouest du pays, la Ruta Lenca (secteur de la mancomunidad (regroupement de municipalités) Colosuca.

\section{La formation et la recherche}

\section{La maîtrise en développement local et tourisme}

Un programme de maîtrise en développement local et tourisme a été préparé dans le cadre du projet. II est articulé autour de quatre composantes. La première assure une base commune de formation à tous les étudiants inscrits au programme; la deuxième porte plus spécifiquement sur le tourisme, la troisième sur l'aménagement territorial compte tenu du tourisme. Une dernière composante concerne la formulation et la réalisation de projets en tourisme. Au terme du programme, les étudiants préparent en groupe une étude de cas. Le programme a reçu l'approbation officielle des autorités responsables de l'enseignement universitaire au Honduras.

Un système sélectif de bourses provenant du projet a permis d'aller chercher en régions des ressortissants locaux, de manière à faciliter leur implication dans le milieu. Au total, le programme a permis à ce jour à 49 étudiants de se graduer. La grande majorité de ceux-ci travaillent dans le domaine dans lequel ils ont été formés. Ils sont très recherchés et très bien accueillis sur le marché du travail, en particulier dans les organismes nationaux et internationaux concernés par le développement des régions structurellement paupérisées et dans leur région d'origine.

\section{La formation de "conseillers accompagnateurs"}

Deux ressources professorales de l'UNITEC ont suivi une session intensive en "accompagnement-conseil » d'initiatives locales auprès de l'organisation SAJE (Service d'aide aux jeunes entrepreneurs) ${ }^{2}$ de Montréal. Ces deux personnes coordonnent à leur tour un groupe de dix diplômés de la maîtrise qui se spécialisent en accompagnement d'initiatives locales dans les

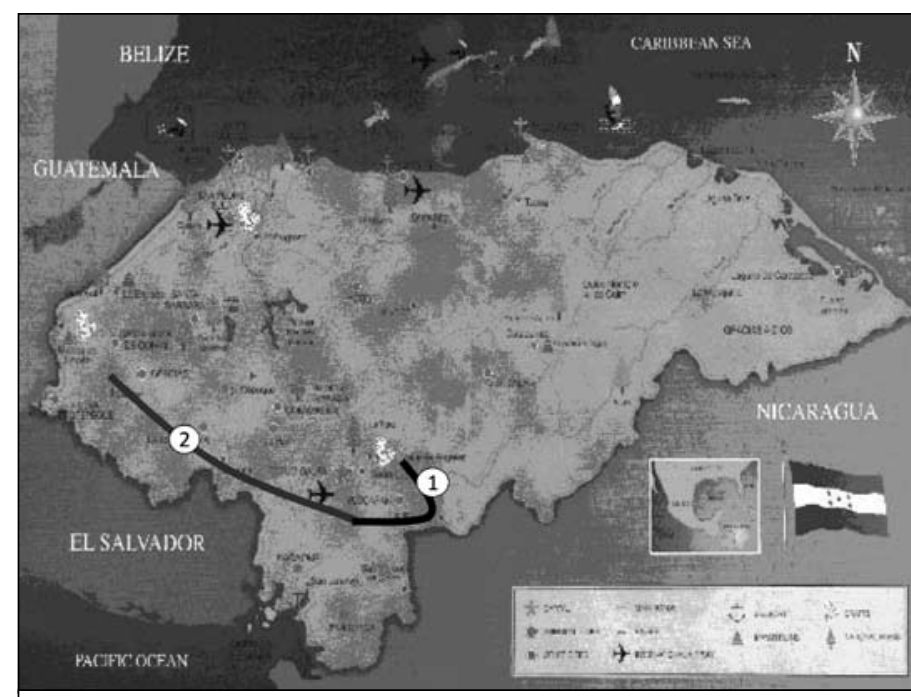

Illustration 3 : Régions cibles du projet.

Source : Projet PUCD volet 2, Développement local durable et tourisme au Honduras.

régions cibles du projet. Les stages de formation d'une durée de quatre mois sont en cours. Une autre ressource professorale de I'UNITEC s'est par ailleurs spécialisée pendant deux ans en "accompagnement-conseil", au sein du projet Fondo Prosperidad (Institut hondurien du tourisme et Banque mondiale ${ }^{3}$ ), destiné à soutenir dans la région de Copán de petites initiatives locales comportant une composante touristique.

\section{Programme de formation de techniciens en développement local de l'Association hondurienne des municipalités (AHMON)}

Le projet a pris en charge les stages des étudiants du programme de l'AHMON. Au total 65 étudiants ont terminé leur stage. Leur travail a permis de monter 12 dossiers sur les ressources touristiques de localités relevant des zones cibles du projet.

\section{Programme de formation pratique (capacitación) dans les deux zones cibles}

À la suite de forums communautaires organisés dans les deux zones cibles, deux programmes de formation, l'un en méthodologie de formulation de projets, l'autre en développement local, ont été montés et offerts dans les régions cibles. Ces programmes, structurés selon des sessions intensives sur plusieurs fins de semaine, ont rejoint au total 80 ressortissants locaux. Le programme de formation se prolonge actuellement par un suivi "d'accompagnement-conseil " de dix initiatives locales. Des étudiants ou des diplômés de la maîtrise en développement local et tourisme ont pris en charge l'organisation pratique et l'enseignement des programmes en communautés et le suivi "d'accompagnement-conseil».

\section{Programme de formation à la recherche}

L'UNITEC a peu développé jusqu'à ce jour son volet recherche. Des sessions de formation aux logiciels EndNote et SPSS ont été 
Organigramme de la maîtrise en développement local et tourisme, UNITEC

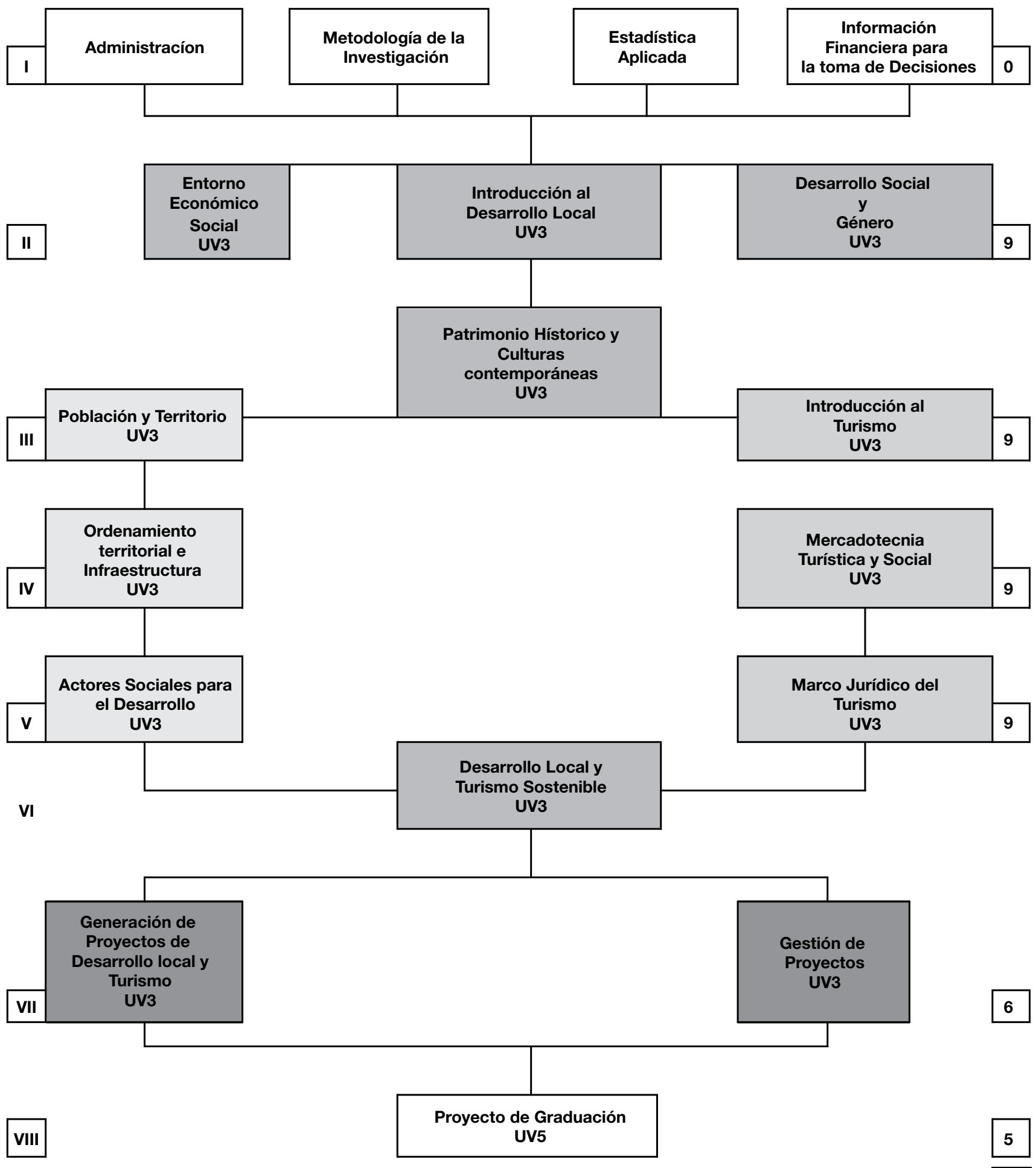


organisées à l'UNITEC et à Montréal (UQAM), à l'intention de professeurs de I'UNITEC associés au projet. Deux documents sont issus de ces formations : un guide EndNote en espagnol permet de systématiser la recherche virtuelle de documentation bibliographique; un autre guide rédigé en espagnol facilite le traitement de données quantitatives à partir de SPSS. Par ailleurs, le livre de Marie-Andrée Delisle et Louis Jolin, Un autre tourisme est-il possible $?^{4}$, a été traduit en espagnol. Ce livre, complété par un dossier présentant des études de cas concernant l'Amérique centrale, servira de référence pédagogique aux étudiants en tourisme.

\section{Doctorat}

Trois ressources professorales de I'UNITEC suivent actuellement un programme de doctorat sur des thèmes associés à la problématique du projet. Le financement de leurs études est partiellement assuré par le projet.

\section{L'infrastructure de soutien}

Pour soutenir l'implication de l'UNITEC auprès des populations locales défavorisées, le projet a organisé au sein de I'UNITEC un Centre de développement local et tourisme (CDLT). Ce dernier poursuit plusieurs objectifs : l'enrichissement de l'information documentaire de l'UNITEC sur les questions de développement local et de tourisme (bases de données, documents spécialisés, guides pédagogiques, bibliothèque virtuelle...), le soutien à la formation sur mesure dans les communautés locales, l'appui à l'organisation de services de consultation et d'assistance technique. La documentation progressivement rassemblée au fil du projet est disponible à la bibliothèque centrale de I'UNITEC, sur support informatique. Le CDLT s'est par ailleurs doté d'un laboratoire informatique équipé d'Internet, d'EndNote (pour la recherche bibliographique) et de logiciels de base (Microsoft Office, SPSS, Arcview pour le traitement cartographique). Le laboratoire informatique est relié à 22 centres informatiques locaux dont l'équipement provient principalement de financements complémentaires, en particulier du projet ICMA-CDLT (International City Management Association, USAID - Centre de développement local et tourisme à I'UNITEC), dont la mise en œuvre a été assurée par le CDLT.

\section{Perspectives d'avenir}

Le temps des semailles touche à sa fin. Comment se profile l'avenir à moyen terme de l'investissement réalisé par le projet? La formation d'un bassin diversifié de ressources humaines spécialisées (maîtrise et doctorat, expertise en accompagnement d'initiatives locales, spécialistes locaux) constitue un apport indéniable du projet. L'accueil des personnes formées sur le marché du travail, y compris dans les communautés d'origine, dans les universités et dans les organisations préoccupées par le développement local et la lutte pour la réduction de la pauvreté, semble très prometteur. L'avenir le confirmera.

La maîtrise en développement local et tourisme répond à un besoin de formation d'une clientèle cible abondante. Au terme de deux ans de fonctionnement «expérimental », le programme doit par contre être évalué. Les travaux des étudiants, en particulier les travaux de recherche de fin d'études, soulèvent un problème d'encadrement. Le financement des études pour les personnes provenant de milieux défavorisés est crucial. L'UNITEC mise sur un étalement de la période d'études pour réduire la pression des frais de scolarité. Une maîtrise en gestion des ressources naturelles et patrimoniales, financée par la coopération espagnole, vient aussi de débuter. Les finalités de cette maîtrise rejoignent partiellement les préoccupations du programme en développement local et tourisme. Cinq personnes formées dans le cadre du PUCD2 UQAM-UNITEC y collaborent à titre d'enseignant et de chercheur.

L'infrastructure du CDLT, en particulier son réseau de centres informatiques locaux associé au laboratoire d'informatique de I'UNITEC, constitue un atout précieux pour apporter un soutien continu aux communautés locales. L'Institut technologique de Monterrey (TEC Monterrey) dispose d'une longue expérience d'intervention auprès de communautés locales défavorisées du Mexique à partir d'une infrastructure similaire. Avec le soutien du projet PUCD2 UQAM-UNITEC, I'UNITEC a pris I'initiative de s'associer au TEC pour adapter le «modèle Monterrey » au contexte hondurien. Le processus d'appropriation à moyen terme des lignes directrices du projet UQAM-UNITEC semble ainsi suivre son cours et prendre progressivement consistance.

Paul Bodson et Jorge Guerrero sont professeurs associés au Département d'études urbaines et touristiques de l'Université du Québec à Montréal.

\section{Notes}

1 Le projet PUCD2 2003-2009 est financé par l'ACDI (Canada).

2 [http://www.sajemontrealmetro.com/].

3 [http://www.politicalperspectives.org.uk/General/Issues/V1-12007/CIP-2007-01-09.pdf].

4 Presses universitaires du Québec, 2007, [http://www.puq.ca/fr/ auteur_fiche.asp?nolntervenant=01399] 